\title{
Identificação de Autor em Manuscritos com Pouco Texto usando Arquiteturas Profundas
}

\author{
Pedro Lins de Araujo Lima* Cleber Zanchettin** \\ * Centro de Informática, Universidade Federal de Pernambuco, PE, \\ (e-mail:plal@cin.ufpe.br). \\ ** Centro de Informática, Universidade Federal de Pernambuco, PE, \\ (e-mail: cz@cin.ufpe.br)
}

\begin{abstract}
This work uses deep neural network-based approaches to address the problem of author identification in short handwritten texts. Machine learning using deep architectures has gained prominence in recent years since it has been very efficient in solving problems that were considered difficult. The problem addressed is not simple and has several applications in society. It involves an open topic in academia, in addition to the restricted number of training examples available. The proposed approach obtained promising results in the databases evaluated.

Resumo: Este projeto utiliza abordagens baseadas em arquiteturas de redes profundas na identificação de autor em textos curtos manuscritos. Esse problema possui várias aplicabilidades na sociedade. A técnica de aprendizagem de máquina utilizando arquiteturas profundas têm ganhado destaque nos últimos anos, pois se mostra muito eficiente na resolução de problemas que se julgavam difíceis. A questão tratada não é simples, pois envolve um tópico em aberto na academia, além da restrita quantidade de exemplos de treinamento disponíveis. A abordagem proposta obteve resultados promissores nas bases de dados avaliadas.
\end{abstract}

Keywords: machine learning; deep learning; author identification; classification; pattern recognition.

Palavras-chaves: aprendizagem de máquina; aprendizagem profunda; identificação de autores; reconhecimento de padrões

\section{INTRODUÇÃO}

O aprendizado de máquina é um método de análise de dados que automatiza o desenvolvimento de modelos de mapeamento de informações baseado em exemplos. Usando algoritmos que aprendem iterativamente a partir de dados, o aprendizado de máquina permite encontrar, automaticamente, padrões nos dados de forma a construir fronteiras de representação e separação com base em exemplos, sejam eles rotulados ou não.

As técnicas de aprendizado de máquina representam o estado da arte na solução de problemas de reconhecimento de padrões, processamento de imagens e extração de informações (Mitchell (1997)). Dentre as técnicas de aprenziado de máquina, a aprendizagem profunda ou deep learning engloba a maior parte dos modelos que têm obtido grandes avanços na solução de diversos problemas, como citado em Bengio (2013) e Srinivas and Babu (2015). Essa técnica corresponde à utilização de uma rede neural com uma grande quantidade de camadas ocultas e permite a captura de informações dos dados com diferentes níveis de abstração e sem a necessidade da extração de características, sendo possível, por exemplo, utilizar como entrada da rede uma imagem ou um texto em vez de um vetor com características da imagem.

$\mathrm{O}$ reconhecimento de texto manuscrito, por sua vez, ainda apresenta vários desafios para os pesquisadores da área, como apresentado por Schmidhuber (2013). O problema de reconhecimento e identificação de autores em textos manuscritos se torna ainda mais desafiador quando se tratam de textos curtos ou com pouca informação manuscrita. Nesse cenário, há muitos trabalhos propostos, porém, normalmente utilizando bases de dados com muitos documentos escritos pelo mesmo autor para o treinamento dos modelos (Fiel and Sablatnig (2015), Louloudis et al. (2011), Louloudis et al. (2013)).

Desta forma, uma das desvantagens dessa abordagem é a necessidade de uma grande base de textos manuscritos redigidos por diferentes autores, bem como vários textos escritos pelo mesmo autor, para que cada um seja identificado corretamente. Isso inviabiliza a utilização destas técnicas em textos curtos, como, por exemplo, na verificação de fraudes no preenchimento de cheques bancários, comparando o padrão de escrita do correntista entre as várias linhas de preenchimento do cheque.

Neste trabalho, buscamos soluções para identificação de autores em textos manuscritos curtos e que permitam obter resultados confiáveis considerando pequenas amostras de treinamento, imagens possivelmente ruidosas e que permitam associações entre informações textuais e numéricas.

Avaliamos duas abordagens utilizando técnicas pertencentes à área de aprendizagem profunda. A primeira foi proposta por Hafemann et al. (2017), que propõe utilizar uma arquitetura profunda como extrator de características e 


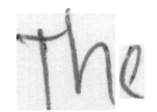

(a)

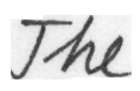

(d)

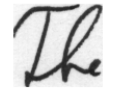

(b)

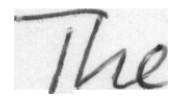

(e)

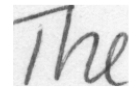

(c)

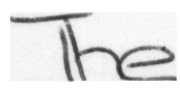

(f)
Figura 1. a-f: Exemplos de imagens do subconjunto c03, cada palavra pertence a um autor diferente.

fazer a classificação com outro algoritmo de aprendizagem de máquina. A segunda abordagem consiste em utilizar a rede profunda para realizar a classificação.

Os experimentos foram realizados com a base de dados IAM Handwritting Database, Marti and Bunke (2002), disponibilizada pela Universidade de Bern. Essa base consiste em diversos formulários nos quais os usuários escreveram determinados textos, formando, assim, um conjunto que permite observar o mesmo formulário escrito por diversos usuários diferentes disponibilizados no formato de texto plano, linhas, frases e palavras.

Optamos por fazer os testes iniciais utilizando o repositório de palavras e, para isso, usamos dois subconjuntos específicos da base de dados, o c03 (com cerca de 4 mil imagens de 6 autores diferentes) e g06 (perto de 12 mil imagens de 17 autores diferentes), os quais facilitam o processo de classificação, visto que em cada palavra está associada a informação do autor que a escreveu. Podemos ver na Figura 1, um exemplo de uma palavra escrita por cada um dos autores do conjunto c03.

Na próxima seção serão apresentados os trabalhos relacionados. Na seção 3 descrevemos nossa proposta. Na seção 4 serão mostrados os resultados obtidos e na última seção as considerações finais.

\section{TRABALHOS RELACIONADOS}

\subsection{Aprendizagem profunda}

A aprendizagem de máquina é um campo da inteligência artificial que lida com a questão de como construir programas de computador que melhoram com a experiência, Mitchell (1997), ou seja, a medida que o algoritmo observa mais exemplos o seu desempenho melhora considerando alguma métrica de avaliação.

Nas últimas décadas uma área da aprendizagem de máquina vem ganhando muito destaque, que é a aprendizagem profunda.

Dentre os modelos baseados em aprendizagem profunda, um dos primeiros trabalhos com reconhecimento na comunidade acadêmica foi apresentado em LeCun et al. (1998), uma rede neural convolucional para realizar a tarefa de reconhecer dígitos manuscritos.

Bengio (2013) examina alguns desafios pertinentes ao contexto da aprendizagem profunda, dentre eles: 1) o pro- blema da escalabilidade dos algoritmos de aprendizagem em relação a modelos e bases de dados; 2) a redução da dificuldade de otimização relacionadas a má formulação do problema ou mínimos locais e 3) o desenvolvimento de procedimentos de inferência mais eficientes.

Em anos mais recentes a evolução dos algoritmos de aprendizagem profunda é notória, principalmente em problemas relacionados a imagens, particularmente o problema de classificação, tendo sido criadas, inclusive, competições para medir o desempenho de novos algoritmos propostos, Russakovsky et al. (2015).

O estado da arte dos métodos de aprendizagem profunda para problemas de classificação muitas vezes é composto de modelos com bom desempenho em competições, como pode ser observado em He et al. (2016).

\subsection{Reconhecimento de textos manuscritos}

O reconhecimento de textos manuscritos é um problema ainda em aberto, que chama a atenção de diversos membros da comunidade acadêmica, como evidenciado em Schmidhuber (2013). Por esse motivo, diversos pesquisadores criaram bases de dados para auxiliar na resolução desse problema, Marti and Bunke (2002), Vargas et al. (2007), Ortega-Garcia et al. (2003), Freitas et al. (2000), entre outros.

Em seu trabalho, Fiel and Sablatnig (2015) propõem um método baseado em redes neurais convolucionais, para identificar o autor de um documento através de uma comparação com uma base de documentos em que os autores são conhecidos; bem como um método para realizar a recuperação, que é a tarefa de encontrar textos com escritas parecidas ou recuperar todos os documentos de um mesmo autor.

O trabalho de Hafemann et al. (2017), que pode ser considerado a maior inspiração para uma das abordagens exploradas neste artigo, utiliza uma rede neural convolucional para extrair as características de imagens de assinaturas que serão posteriormente classificadas para decidir se a assinatura fornecida é autêntica ou uma fraude.

\section{ABORDAGENS AVALIADAS}

Neste trabalho foram investigadas duas abordagens: a primeira com base em Hafemann et al. (2017), que consiste em utilizar uma rede neural profunda para extrair as características das imagens e posteriormente fazer a classificação utilizando um classificador diferente; e a segunda, por sua vez, consiste em utilizar redes profundas com arquiteturas diferentes para realizar a classificação das imagens.

\subsection{Abordagem de extração de características}

Inicialmente aplicamos a abordagem proposta em Hafemann et al. (2017) para a classificação de assinaturas, utilizando o conceito de random forgeries, que são assinaturas de outras pessoas, e de skilled forgeries, que são tentativas de forjar a assinatura de determinada pessoa; com o objetivo de verificar se a assinatura é real ou uma tentativa de cópia. Para isso, os autores utilizaram as bases de assinaturas: GPDS (Vargas et al. (2007)), MCYT (Ortega-Garcia 
Table 1: Summary of the CNN layers

\begin{tabular}{lcc}
\multicolumn{3}{c}{ Table 1: Summary of the CNN layers } \\
\hline Layer & Size & Other Parameters \\
\hline Input & $1 \times 150 \times 220$ & \\
Convolution (C1) & $96 \times 11 \times 11$ & stride $=4$, pad=0 \\
Pooling & $96 \times 3 \times 3$ & stride $=2$ \\
Convolution (C2) & $256 \times 5 \times 5$ & stride $=1$, pad=2 \\
Pooling & $256 \times 3 \times 3$ & stride $=2$ \\
Convolution (C3) & $384 \times 3 \times 3$ & stride $=1$, pad=1 \\
Convolution (C4) & $384 \times 3 \times 3$ & stride $=1$, pad=1 \\
Convolution (C5) & $256 \times 3 \times 3$ & stride $=1$, pad=1 \\
Pooling & $256 \times 3 \times 3$ & stride $=2$ \\
Fully Connected (FC6) & 2048 & \\
Fully Connected (FC7) & 2048 & \\
Fully Connected + Softmax $(P(\mathbf{y} \mid X))$ & M & \\
Fully Connected + Sigmoid $(P(f \mid X))$ & 1 & \\
\hline
\end{tabular}

Figura 2. Arquitetura da rede SigNet, esta tabela foi adaptada de Hafemann et al. (2017)

et al. (2003)), CEDAR (Kalera et al. (2004)), e PUC-PR (Freitas et al. (2000)). O modo como a rede obtém as características é eliminando a camada de decisão, fazendo com que a saída da rede seja o vetor de características dos exemplos apresentados a ela. Esse vetor que é utilizado como entrada para um outro classificador que vai tomar a decisão a cerca da classe associada ao exemplo de teste.

Adaptando a abordagem de Hafemann et al. (2017) ao problema de identificação de autor em manuscritos com pouco texto, os exemplos não consistem de assinaturas e sim de textos curtos, desta forma, utilizamos a base $I A M$ (Marti and Bunke (2002)), porém mantivemos a estratégia de usar a rede SigNet para a extração das características das imagens. Nesse caso, utilizamos a saída da camada anterior a primeira camada Fully Connected + Softmax na Figura 2, bem como o pré-processamento fornecido pelo código dos autores, mudando apenas o parâmetro canvas size, responsável por representar o tamanho da janela em que a imagem da palavra é centralizada e o ruído, caso exista, será removido. Após o pré-processamento das imagens e a extração das características, utilizamos os vetores obtidos como entrada para o classificador SVM, proposto por Cortes and Vapnik (1995), visto que foi o classificador que obteve o melhor desempenho no artigo original.

Os experimentos consistiram de extrair as características de todas as palavras do conjunto, totalizando 2.048 características por palavra. Em seguida, todos os vetores de características foram armazenados em um dataframe, que foi dividido em 30\% dos exemplos para teste e $70 \%$ para o treinamento do modelo de classificação. Durante o treinamento foi utilizado o método de $K$-Fold cross validation, com $k=5$, de modo que a divisão em teste e treino foi feita 5 vezes usando partições diferentes do conjunto e no final uma média do desempenho em cada uma das 5 vezes considerada.

\subsection{Abordagem de classificação direta}

A segunda abordagem investigada foi mais objetiva, pois utilizamos a própria rede profunda para a classificação das instâncias, diferentemente da abordagem anterior. Para isso, usamos a biblioteca fastai ${ }^{1}$, também de Python e que possui diversas arquiteturas de redes profundas prétreinadas com o conjunto de imagens ImageNet, apresentado em Deng et al. (2009).

Nessa abordagem, os experimentos consistiram em realizar a classificação de cada um dos subconjuntos escolhidos da base (c03 e g06) e também da união dos dois utilizando diferentes arquiteturas de redes profundas disponibilizadas pela biblioteca. Além dos subconjuntos puros, também testamos a classificação realizando data augmentation através de rotação, espelhamento e aplicação de zoom nas imagens, ou seja, para cada época de treinamento uma nova transformação na imagem é realizada envolvendo essas transformações, portanto, o modelo utiliza o mesmo número de imagens em cada época, que é o mesmo dos dados de treinamento originais, mas ele utiliza versões diferentes das imagens, fazendo com que o range de imagens diferentes que o modelo utiliza no aprendizado aumente a cada época. Logo, seja $N$ o número de imagens nos dados e $E$ o número de épocas de treinamento, a rede recebe $N x E$ imagens no total.

Testamos quatro arquiteturas de redes profundas diferentes dentre as disponibilizadas pela biblioteca, sendo duas da família ResNet, apresentada em He et al. (2016) e duas da família $V G G$, apresentada em Simonyan and Zisserman (2014). As duas arquiteturas ResNet (ou Residual Network, que recebe esse nome devido ao conceito de residual connections, ou skip connections, que tem como objetivo diminuir o tempo de treinamento da rede e aumentar sua profundidade), utilizadas foram a ResNet34 e ResNet50. Essas redes, diferentemente de outros modelos como as redes VGG que serão citadas a seguir, não intercalam camadas de convolução com camadas de convolução + max pooling, em vez disso elas possuem uma camada de average pooling global e uma camada fully connected com ativação Softmax no final de sua arquitetura, sendo o restante formado por camadas de convolução com filtros, em geral, de dimensões $3 \times 3$. É por esse motivo que é possível fazer as residual connections, já que elas precisam ser feitas em camadas cuja entrada e saída possuem as mesmas dimensões, e como essas redes utilizam padding nas camadas em que a entrada e saída são de dimensões diferentes, essa propriedade se mantém, sendo possível a utilização dessas conexões. Dependendo da arquitetura da rede, os blocos formados pelas residual connections podem ser de duas ou três camadas, sendo os de duas mais comuns em arquiteturas menores como a 18 ou 34 e os de três em arquiteturas maiores, como 50, 101 e 152. Podemos ver em mais detalhes as residual connections e seus blocos na Figura 3.

É possível observar a arquitetura ResNet34 em relação a uma rede profunda comum de 34 camadas na Figura 4, na qual fica evidente a presença das residual (skip) connections. É importante ressaltar que a eficácia da rede do tipo ResNet para resolver problemas de classificação de imagens foi comprovada na competição ILSVRC, Russakovsky et al. (2015) (ImageNet Large Scale Visual Recognition Challenge), a qual foi a arquitetura vencedora na edição de 2015.

\footnotetext{
1 https://github.com/fastai/fastai
} 

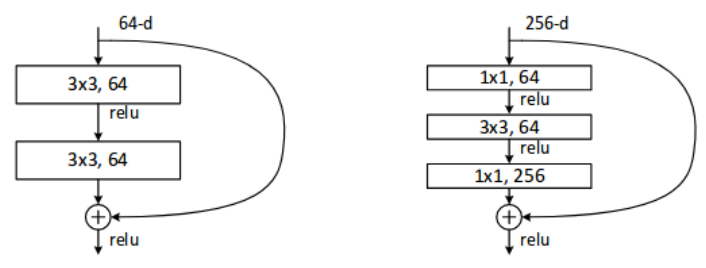

Figura 3. Exemplos de residual connections e os blocos formados por essas conexões. Disponível em: http://bit.ly/30d10om
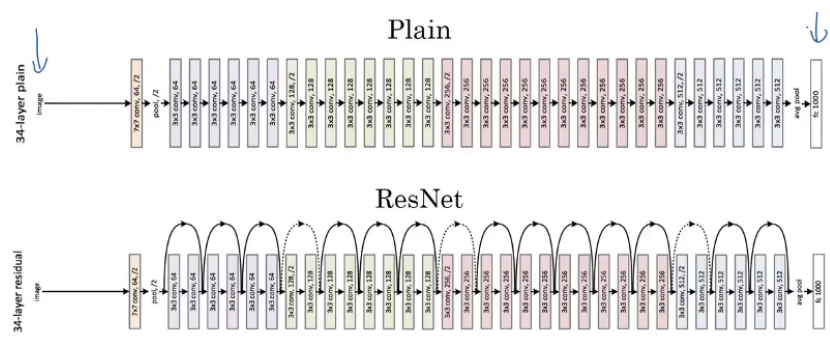

Figura 4. Arquitetura ResNet34 em relação a arquitetura profunda "plain"com 34 camadas. Disponível em: http://bit.ly/2YXLiAV

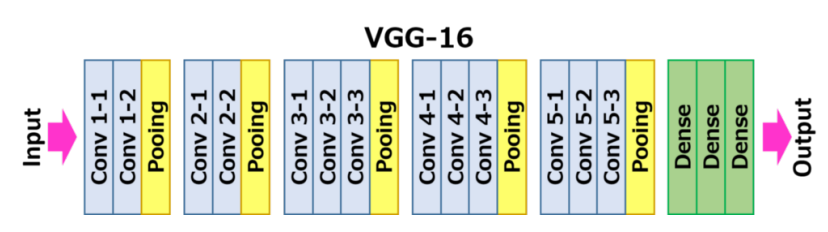

Figura 5. Arquitetura das redes VGG. Disponível em: http://bit.ly/33T28Qq

Já as arquiteturas que fazem parte da família $V G G$ foram a $V G G 16$ e $V G G 19$, que são redes profundas mais simples nos quais não há muitas inovações em relação aos modelos originais de redes profundas, como a LeNet proposta em LeCun et al. (1998), além do aumento do número de camadas (a LeNet mais conhecida possui 5 camadas enquanto a VGG original tem 16). Dessa forma, as redes do tipo VGG são redes contendo 13 camadas intercalando as operações de convolução e operações de convolução + max pooling, seguidas de duas camadas fully connected e uma camada de saída com ativação Softmax com 1000 nós. Só essa mudança já surtiu grande efeito na eficiência das redes profundas, visto que as redes VGG apresentam resultados ainda melhores do que os primeiros modelos de redes profundas como a LeNet. Podemos ver a estrutura das redes VGG na Figura 5

A Figura-5 apresenta a estrutura da rede $V G G 16$ que é muito semelhante à estrutura da rede profunda que vemos na Figura 4 sendo comparada com a rede ResNet, evidenciando o fato de que esse tipo de arquitetura, apesar de apresentar ótimos resultados para diversos problemas da atualidade, não apresenta muitas diferenças das redes profundas originais, além do aumento do número de camadas.

Os experimentos dessa abordagem, como dito anteriormente, foram feitos utilizando redes pré-treinadas, ou seja, com os pesos congelados de um treinamento anterior, de modo que é necessário descongelar esses pesos para que a rede se adapte melhor aos dados do problema em questão. $\mathrm{O}$ treinamento foi feito tanto utilizando data augmentation, quanto sem utilizar essa técnica e durou 7 épocas, testando 3 learning rates diferentes, com a acurácia de testes calculada ao final do treinamento.

\subsection{Avaliação dos resultados}

A avaliação dos resultados foi feita, majoritariamente, em paralelo à implementação das abordagens e realização dos testes. Em sua maior parte, para avaliar os resultados, utilizamos a métrica de acurácia (accuracy), tanto para avaliar os modelos treinados utilizando a primeira abordagem, quanto para avaliar os modelos pré-treinados utilizados na segunda abordagem.

É importante salientar que, apesar de não ser a métrica mais confiável para avaliar o desempenho de um classificador, a acurácia ainda é uma métrica muito popular em diversos problemas de diversas áreas, sendo também uma métrica de fácil entendimento para pessoas que não possuem conhecimento das métricas específicas para a avaliação do desempenho de classificadores.

\section{RESULTADOS E DISCUSSÃO}

\subsection{Resultados e discussão da primeira abordagem}

Para a primeira abordagem, utilizando a arquitetura profunda como extrator de características das imagens das palavras fornecidas, usamos majoritariamente o conjunto c03. O treinamento e os testes foram realizados com o classificador SVM, o qual obteve os melhores resultados no artigo original. Esse conjunto foi escolhido para análise inicial pois queríamos comparar os resultados obtidos com os resultados iniciais obtidos pela outra abordagem explorada no mesmo conjunto. $\mathrm{O}$ que se verificou nos resultados foi uma forte dependência do parâmetro canvas size no pré-processamento dos dados, o qual precisou ser bastante alterado em relação ao valor padrão para se obter resultados satisfatórios, como podemos observar na Tabela 1

Tabela 1. Acurácia da abordagem de extração de características.

\begin{tabular}{cc} 
Dimensões (canvas size) & Acurácia \\
\hline $952 \times 1360$ & $75.94 \%$ \\
$600 \times 1000$ & $85.68 \%$ \\
$150 \times 750$ & $93.36 \%$ \\
$175 \times 850$ & $93.38 \%$ \\
$150 \times 825$ & $93.47 \%$ \\
\hline
\end{tabular}

Pode ser verificado que a acurácia do classificador foi muito dependente do parâmetro canvas size utilizado no pré-processamento das palavras antes da extração de características. O ajuste no parâmetro foi feito de forma empírica, partindo de uma dimensão base, representada pela primeira linha da Tabela 1 e fazendo decrementos primeiro na altura no sentido em que a acurácia aumentava, e depois na largura no mesmo sentido, demandando muitos experimentos para chegar a resultados satisfatórios para esse conjunto. Isso nos levou a pensar se esse ajuste no parâmetro não estaria causando overfitting no modelo, ou seja, o modelo estava sendo enviesado para responder bem ao conjunto de dados que apresentamos para ele, mas 
podia não responder tão bem para exemplos que não foram vistos em treinamento.

Para verificar se isso realmente estava acontecendo resolvemos utilizar o subconjunto g06 da base $I A M$ com o mesmo classificador para obter os resultados da tabela anterior e avaliando a tupla de valores de canvas size que apresentou melhor resultado nos testes anteriores, ou seja, $(150,825)$ e o resultado da acurácia foi de cerca de $73 \%$, evidenciando que o valor do parâmetro é muito dependente do conjunto que está sendo utilizado para treinar e realizar a classificação, e que o resultado inicialmente positivo de $93.47 \%$ obtido através de um overfitting em que o classificador ficou muito especializado nos dados do conjunto com o qual foi treinado.

\subsection{Resultados e discussão da segunda abordagem}

Como explicado na seção anterior, para a segunda abordagem, em vez de usarmos as redes profundas para apenas extrair as características das imagens das palavras e o SVM para realizar a classificação, as redes profundas foram utilizadas como o próprio classificador. Dessa forma, a rede recebia como entrada a imagem e retornava como saída a qual autor a palavra da imagem pertencia. Para isso, foi utilizada a biblioteca fastai que disponibiliza modelos de redes profundas pré-treinados.

Foram testadas quatro arquiteturas profundas diferentes: a ResNet34, ResNet50, VGG16 e VGG19. Os testes foram feitos utilizando o conjunto c03, g06 e a união dos dois conjuntos. Além disso, realizamos os testes usando data augmentation e também sem utilizar essa técnica, que consistiu de rotacionar, espelhar e aplicar zoom nas imagens.

Tabela 2. Acurácia da classificação no conjunto $\mathrm{c03}$

\begin{tabular}{ccc} 
Arquitetura & c03 & c03 com data augmentation \\
\hline ResNet34 & $97.36 \%$ & $97.59 \%$ \\
ResNet50 & $97.36 \%$ & $97.70 \%$ \\
VGG16 & $98.05 \%$ & $97.25 \%$ \\
VGG19 & $97.93 \%$ & $98.05 \%$ \\
\hline
\end{tabular}

Podemos observar na Tabela 2 os resultados de acurácia para cada uma das quatro arquiteturas no conjunto c03 com e sem data augmentation, observamos que não há grande diferença nos resultados, seja entre os classificadores ou nos resultados com e sem data augmentation. Esse conjunto foi o que obteve os melhores resultados dentre todos os avaliados, visto que era o conjunto com o menor numero de exemplos e classes, cerca de 4 mil exemplos divididos entre 6 classes (autores) diferentes. Provavelmente por isso todas as arquiteturas tiveram um desempenho muito bom na classificação, especialmente as da família $V G G$, embora a diferença não tenha sido muito grande em relação às ResNet.

Tabela 3. Acurácia da classificação no conjunto g06

\begin{tabular}{ccc} 
Arquitetura & g06 & g06 com data augmentation \\
\hline ResNet34 & $94.10 \%$ & $94.49 \%$ \\
ResNet50 & $94.36 \%$ & $94.49 \%$ \\
VGG16 & $94.83 \%$ & $94.79 \%$ \\
VGG19 & $94.83 \%$ & $94.36 \%$ \\
\hline
\end{tabular}

Ao observar na Tabela 3 , os resultados das diferentes arquiteturas no conjunto g06, que possui cerca de 12 mil instâncias de palavras pertencentes a 17 autores diferentes, podemos ver que o desempenho das redes na classificação, que já era muito próximo no conjunto anterior, ficou ainda mais nesse conjunto. É importante salientar também que a queda que ocorreu no desempenho pode ser resultado do número muito maior de exemplos observados nesse conjunto, que tem quase o triplo de tamanho do conjunto anterior.

Tabela 4. Acurácia da classificação no conjunto
\[ \mathrm{c03}+\mathrm{g} 06 \]

\begin{tabular}{ccc} 
Arquitetura & c03+g06 & c03+g06 com data augmentation \\
\hline ResNet34 & $94.49 \%$ & $94.59 \%$ \\
ResNet50 & $94.34 \%$ & $94.49 \%$ \\
VGG16 & $95.18 \%$ & $95.37 \%$ \\
VGG19 & $94.90 \%$ & $95.40 \%$ \\
\hline
\end{tabular}

A Tabela 4 apresenta os resultados obtidos pelos diferentes modelos de rede profunda na união dos dois conjuntos que foram testados anteriormente, totalizando cerca de 16 mil exemplos com 23 classes diferentes, na qual cada classe corresponde a um autor dentre os que faziam parte dos dois conjuntos anteriores. Podemos observar que, novamente, o desempenho de todos os classificadores foi muito próximo, com um leve destaque para as redes da família $V G G$, que obtiveram um resultado um pouco melhor do que as ResNet. Isso pode ter acontecido pelo fato de o número de exemplos, apesar de parecer, não ser muito grande (a competição ILSVRC, por exemplo, possui 150 mil imagens divididas entre mil categorias, pelo menos até o ano de 2015, quando a ResNet foi a vencedora), e isso pode favorecer a arquitetura mais simples das redes $V G G$, enquanto a ResNet poderia ter um desempenho melhor caso houvessem mais dados, como por exemplo, se tivéssemos utilizado a base $I A M$ completa, o que não foi feito pois precisava ser realizado um processamento sobre o arquivo de metadados fornecido, mas que na tentativa de realizar esse processamento não conseguimos observar quantos autores existem no total, por isso decidimos manter os testes sobre os conjuntos c03 e g06 que informam no próprio nome dos arquivos das imagens qual é o autor que escreveu.

É importante notar também que o número de camadas não teve muita influência no resultado da avaliação dos modelos, visto que as redes ResNet tiveram o desempenho muito parecido em todos os conjuntos testados apesar de uma ter 34 camadas e a outra 50. Isso também aconteceu com as redes $V G G$, visto que uma das arquiteturas possui 16 camadas e a outra 19, mesmo assim os resultados foram bastante parecidos entre elas.

\section{CONCLUSÃO}

A partir dos resultados obtidos e discutidos na seção anterior, podemos observar que as técnicas de aprendizagem profunda realmente se mostraram eficazes para avançar nas tentativas de resolução do problema de identificação de autor em textos curtos manuscritos. Os resultados obtidos podem ser considerados satisfatórios, apesar de não termos nos aprofundado muito na questão do pré-processamento dos dados e também no fine tuning dos hiperparâmetros 
envolvidos nas arquiteturas testadas (principalmente durante os testes da segunda abordagem). Portanto, isso nos leva a acreditar que se mais pesquisas neste tema forem realizadas, dando mais atenção a esses elementos, os resultados poderão ser ainda melhores.

A primeira abordagem, por se tratar de uma adaptação de um outro problema, apesar de bons resultados, se mostrou mais limitada, visto que usamos apenas a rede profunda fornecida pelos autores do artigo para extrair as características. Além disso, os resultados foram muito dependentes do pré-processamento realizado, principalmente do parâmetro canvas size, posto que a mudança nesse parâmetro resultou em uma grande discrepância entre os resultados para um mesmo conjunto de dados. Se muita atenção for dada a esse parâmetro em especial, pode causar overfitting, de modo que o sistema não faria uma boa generalização para novos exemplos, como foi mostrado na seção anterior.

Na segunda abordagem tivemos mais liberdade para experimentar diversos tipos de arquitetura na classificação dos exemplos de texto, por isso obtivemos melhores resultados, mesmo sem termos nos aprofundado no pré-processamento das imagens, o que leva a crer que resultados ainda melhores podem ser obtidos se for dada maior atenção a essa etapa do pipeline de classificação, bem como nos hiperparâmetros específicos de cada uma das arquiteturas testadas. Em decorrência dessa flexibilidade, tanto para a escolha da arquitetura quanto para a utilização ou não de pré-processamento, data augmentation, entre outros elementos, julgamos que esse tipo de abordagem se mostrou mais promissora que sua predecessora.

\section{AGRADECIMENTOS}

Agradeço a Camila, Fernanda, Nadja e Jorge, pelo apoio incondicional e por acreditarem em mim; ao professor Cleber, pela orientação e confiança; aos meus amigos, pelo incentivo e ajuda; ao CIn e à UFPE, pela estrutura fornecida; e ao CNPq pelo apoio fundamental.

\section{REFERENNCIAS}

Bengio, Y. (2013). Deep learning of representations: Looking forward. In International Conference on Statistical Language and Speech Processing, 1-37. Springer.

Cortes, C. and Vapnik, V. (1995). Support-vector networks. Machine learning, 20(3), 273-297.

Deng, J., Dong, W., Socher, R., Li, L.J., Li, K., and Fei-Fei, L. (2009). Imagenet: A large-scale hierarchical image database. In 2009 IEEE conference on computer vision and pattern recognition, 248-255. Ieee.

Fiel, S. and Sablatnig, R. (2015). Writer identification and retrieval using a convolutional neural network. In G. Azzopardi and N. Petkov (eds.), Computer Analysis of Images and Patterns, 26-37. Springer International Publishing, Cham.

Freitas, C., Morita, M., de Oliveira, L.E.S., Justino, E., El Yacoubi, A., Lethelier, E., Bortolozzi, F., and Sabourin, R. (2000). Bases de dados de cheques bancários brasileiros.

Hafemann, L.G., Sabourin, R., and Oliveira, L.S. (2017). Learning features for offline handwritten signature verification using deep convolutional neural networks. Pattern Recognition, 70, 163-176.
He, K., Zhang, X., Ren, S., and Sun, J. (2016). Deep residual learning for image recognition. In Proceedings of the IEEE conference on computer vision and pattern recognition, 770-778.

Kalera, M.K., Srihari, S., and Xu, A. (2004). Offline signature verification and identification using distance statistics. International Journal of Pattern Recognition and Artificial Intelligence, 18(07), 1339-1360.

LeCun, Y., Bottou, L., Bengio, Y., Haffner, P., et al. (1998). Gradient-based learning applied to document recognition. Proceedings of the IEEE, 86(11), 22782324.

Louloudis, G., Gatos, B., Stamatopoulos, N., and Papandreou, A. (2013). Icdar 2013 competition on writer identification. In 2013 12th International Conference on Document Analysis and Recognition, 1397-1401. IEEE.

Louloudis, G., Stamatopoulos, N., and Gatos, B. (2011). Icdar 2011 writer identification contest. In 2011 International Conference on Document Analysis and Recognition, 1475-1479. IEEE.

Marti, U.V. and Bunke, H. (2002). The iam-database: an english sentence database for offline handwriting recognition. International Journal on Document Analysis and Recognition, 5(1), 39-46.

Mitchell, T.M. (1997). Machine Learning. McGraw-Hill, Inc., New York, NY, USA, 1 edition.

Ortega-Garcia, J., Fierrez-Aguilar, J., Simon, D., Gonzalez, J., Faundez-Zanuy, M., Espinosa, V., Satue, A., Hernaez, I., Igarza, J.J., Vivaracho, C., et al. (2003). Mcyt baseline corpus: a bimodal biometric database. IEE Proceedings-Vision, Image and Signal Processing, 150(6), 395-401.

Russakovsky, O., Deng, J., Su, H., Krause, J., Satheesh, S., Ma, S., Huang, Z., Karpathy, A., Khosla, A., Bernstein, M., et al. (2015). Imagenet large scale visual recognition challenge. International journal of computer vision, $115(3), 211-252$.

Schmidhuber, J. (2013). Handwritting recognition. http://people.idsia.ch/ ${ }^{\sim}$ juergen/handwriting. html. Accessed: 2016-08-30.

Simonyan, K. and Zisserman, A. (2014). Very deep convolutional networks for large-scale image recognition. arXiv preprint arXiv:1409.1556.

Srinivas, S. and Babu, R.V. (2015). Learning the architecture of deep neural networks. CoRR, abs/1511.05497.

Vargas, F., Ferrer, M., Travieso, C., and Alonso, J. (2007). Off-line handwritten signature gpds-960 corpus. In Ninth International Conference on Document Analysis and Recognition (ICDAR 2007), volume 2, 764-768. IEEE. 